\title{
SORET AND DUFOUR EFFECTS ON MHD MICROPOLAR FLUID FLOW OVER A LINEARLY STRETCHING SHEET, THROUGH A NON -DARCY POROUS MEDIUM
}

\author{
G.V.R. REDDY* and Y.H. KRISHNA \\ Department of Mathematics, K L E F \\ Vaddeswaram, Guntur, AP, INDIA - 520522 \\ E-mail: gvrr1976@kluniversity.in
}

\begin{abstract}
In this paper, we discuss the Soret and Dufour effects on an MHD micropolar fluid flow over a linearly stretching sheet, through a non-Darcy porous medium, where stretching velocity of the sheet varies linearly with distance from the origin, and, temperature and concentration vary non-linearly in the boundary layer region. By suitable similarity transformations, the governing boundary layer equations are transformed to ordinary differential equations. These equations are solved by numerical computations with bvp4c along with the shooting technique method. The effects of the magnetic parameter, Soret number and Dufour number on velocity profiles, microrotation profile, heat transfer, and concentration, skin- friction, Nusselt number and Sherwood number are computed, discussed and analysed numerically and presented through tables and graphs.
\end{abstract}

Key words: MHD flow, magnetic parameter, Dufour number, Soret number.

\section{Introduction}

The fluid model introduced by Eringen [1] exhibits some microscopic effects arising from the local structure and micro-motion of the fluid elements. The model of a micropolar fluid represents fluids consisting of rigid, randomly oriented (or spherical) particles suspended in a viscous medium where the deformation of the particles is ignored. Micropolar fluids have been shown to accurately simulate the flow characteristics of polymeric additives, geo-morphological sediments, colloidal suspensions, hematological suspensions, liquid crystals, lubricants, etc. Sakiadis [2] was the first to study the flow field due to a surface which is moving with a constant velocity in a quiescent fluid. The dynamics of the boundary layer flow over a stretching surface originated from the pioneering work of Crane [3]. The discussion and applications of convective transport in porous media can be found in the book by Nield and Bejan [4]. The mathematical theory of equations of micropolar fluids and applications of these fluids in the theory of lubrication and in the theory of porous media are presented by Lukaszewicz [5]. Srinivasacharya et al. [6] analyzed the flow and heat and mass transfer characteristics of free convection on a vertical plate with variable wall temperature and concentration in a doubly stratified micropolar fluid. Ramachandran et al. [7] discussed the effect of the Prandtl number on the linear stability of a compressible Couette flow. Asma Khalid et al. [8] analyzed the conjugate transfer of heat and mass in unsteady flow of a micropolar fluid with wall couple stress. Nadeem et al. [9] considered the optimal and numerical solutions on an MHD micropolar nanofluid between rotating horizontal parallel plates. Das [10] discussed slip effects on heat and mass transfer in an MHD micropolar fluid flow over an inclined plate with thermal radiation and chemical reaction. Adhikari et al. [11] discussed an MHD micropolar fluid flow towards a vertical surface in the presence of heat flux. Habibi et al. [12] analysed the mixed convection MHD flow of a nanofluid over a non-linear stretching sheet with effects of viscous dissipation and variable magnetic field. Chaudhary et al. [13] discussed the effect of chemical reaction on an MHD micropolar fluid flow past a vertical plate in slip-flow regime. Ali Kashif et al. [14] analysed the numerical simulation of an unsteady water-based nanofluid flow and heat transfer

\footnotetext{
${ }^{*}$ To whom correspondence should be addressed
} 
between two orthogonally moving porous coaxial disks. Mohyuddin et al. [15] studied the optimal and numerical solutions of an MHD flow of a radiative micropolar nanofluid in a porous channel. El-Dabe et al. [16] analysed the numerical solution of an MHD flow of a micropolar fluid with heat and mass transfer towards a stagnation point on a vertical plate. Srinivas Maripala et al. [17] discussed the MHD effects on a micropolar nanofluid flow over a radiative stretching surface with thermal conductivity. Ali Chamkha et al. [18] analysed the unsteady MHD natural convection from a heated vertical porous plate in a micropolar fluid with Joule heating, chemical reaction and radiation effects. Sandeep [19] studied the unsteady boundary layer flow of a thermophoretic MHD nanofluid past a stretching sheet with space and time dependent internal heat source/sink. Khedr [20] analysed the MHD flow of a micropolar fluid past a stretched permeable surface with heat generation or absorption. Sandeep et al. [21] discussed the dual solutions for an unsteady mixed convection flow of MHD micropolar fluid over a stretching/shrinking sheet with nonuniform heat source/sink. Satya Narayana et al. [22] studied the effects of Hall current and radiation absorption on an MHD micropolar fluid in a rotating system. Kelson [23] analysed the effects of surface conditions on flow of a micropolar fluid driven by a porous stretching sheet. Reddy et al. [24] studied the MHD mixed convection oscillatory flow over a vertical surface in a porous medium with chemical reaction and thermal radiation. Pal et al. [25] analyzed the effects of viscous-Ohmic dissipation and variable thermal conductivity on a steady two-dimensional hydromagnetic flow, heat and mass transfer of a micropolar fluid over a stretching sheet embedded in a non-Darcian porous medium with non-uniform heat source/sink and thermal radiation. In the above work the Soret and Dufour effects on an MHD micropolar fluid flow over a non-Darcy porous medium was not studied. Lakshmi et al. [26] studied the thermal radiation and variable viscosity on steady MHD free convective flow over a stretching sheet in the presence of heat source, dissipation and chemical reaction. Jhansi Rani et al. [27] analysed the heat and mass transfer effects on an MHD free convection flow over an inclined plate embedded in a porous medium. Gorla et al. [28] studied the radiation and chemical reaction effects on an MHD flow along a moving vertical porous plate.

In this paper, we discuss the Soret and Dufour effects on an MHD micropolar fluid flow over a linearly stretching sheet, through a non-Darcy porous medium, where stretching velocity of the sheet varies linearly with distance from the origin, and, temperature and concentration vary non-linearly in the boundary layer region. By suitable similarity transformations, the governing boundary layer equations are transformed to ordinary differential equations and these equations are solved by numerical computations with bvp4c with the shooting technique method. The effects of the magnetic parameter, Soret number and Dufour number on velocity profiles, microrotation profile, heat transfer, and concentration, skin- friction, Nusselt number and Sherwood number are computed, discussed and analysed numerically and presented through tables and graphs.

\section{Mathematical formulation of the problem}

We consider a steady two-dimensional mixed convection flow of an incompressible, electrically conducting micropolar fluid towards a surface coinciding with the plane $y=0$ and the flow region $y>0$. The $x$-axis is taken in the direction along the motion of the sheet and the $y$-axis is taken perpendicular to it. The flow is generated by the action of two equal and opposite forces along the $x$-axis and the sheet is stretched in such a way that the velocity at any instant is proportional to the distance from the origin $(x=0)$. Further, the flow field is exposed to the influence of an external transverse magnetic field of strength $B=\left(0, B_{0}, 0\right)$. It is assumed that the whole size of porous plate is taken constant. The non-Darcian inertia effect is considered in the model. The thermal conductivity of the fluid is assumed to be functions of temperature. There is a first order chemical reaction between the diffusing species and the fluid. The effect of thermal radiation, thermo-diffusion and diffusion-thermo are considered. The Reynolds number is assumed to be small. The pressure gradient, body forces, Hall currents, frictional heating due to viscous dissipation and Ohmic heating due to the application of the magnetic field are negligible and hence not considered in the present model. 
The temperature and concentration of the plate surface is always greater than their free stream values. The flow configuration and the coordinate system are shown in Fig.1.

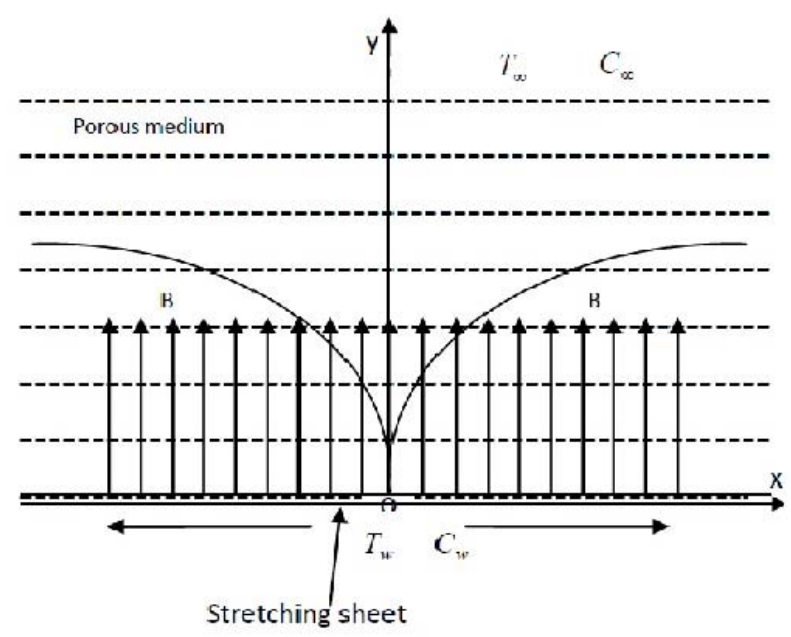

Fig.1. Physical flow model over a stretching surface.

Under the foregoing assumptions and invoking the usual Boussinesq approximation, the governing equations that describe the physical situation can be written as follows:

The continuity equation

$$
\frac{\partial u}{\partial x}+\frac{\partial v}{\partial y}=0
$$

the equation of momentum

$$
\begin{aligned}
& u \frac{\partial u}{\partial x}+v \frac{\partial u}{\partial y}=\left(\frac{\mu+\kappa_{V}}{\rho}\right) \frac{\partial^{2} u}{\partial y^{2}}+\left(\frac{\kappa_{V}}{\rho}\right) \frac{\partial N}{\partial y}+g \beta_{T}\left(T-T_{\infty}\right)+ \\
& +g \beta_{C}\left(C-C_{\infty}\right)-\frac{\sigma B_{0}^{2}}{\rho} u-\frac{v}{k_{p}} u^{\prime}-\frac{C_{E}}{\sqrt{k_{p}}} u^{2}
\end{aligned}
$$

angular momentum equation

$$
u \frac{\partial N}{\partial x}+v \frac{\partial N}{\partial y}=\left(\frac{\gamma}{\rho j}\right) \frac{\partial^{2} N}{\partial y^{2}}-\left(\frac{\kappa_{V}}{\rho j}\right)\left(2 N+\frac{\partial u}{\partial y}\right),
$$

energy equation

$$
u \frac{\partial T}{\partial x}+v \frac{\partial T}{\partial y}=\frac{1}{\rho C_{p}} \frac{\partial}{\partial y}\left(k \frac{\partial T}{\partial y}\right)-\frac{1}{\rho C_{p}} \frac{\partial q_{r}}{\partial y}+\left(\frac{D_{M} K_{T}}{C_{S} C_{P}}\right) \frac{\partial^{2} C}{\partial y^{2}},
$$

mass diffusion equation 


$$
u \frac{\partial C}{\partial x}+v \frac{\partial C}{\partial y}=D_{M} \frac{\partial^{2} C}{\partial y^{2}}-k_{c}\left(C-C_{\infty}\right)+\left(\frac{D_{M} K_{T}}{T_{M}}\right) \frac{\partial^{2} T}{\partial y^{2}}
$$

where $u$ and $v$ are velocity components along the $x$ and $y$ axes, respectively; $\kappa_{v}$ is the micro-rotation viscosity or the vortex viscosity; $j$ is the micro-inertia density; $\mu$ is the dynamic viscosity; $v$ is the kinematic viscosity. $N$ is the microrotation component normal to the $x-y$ plane; $\gamma$ is the spin gradient viscosity; $\sigma$ is the electrical conductivity of the fluid and is assumed to be constant; $k_{p}$ is permeability of the porous medium; $C_{E}$ is the form of drag coefficient which is independent of viscosity and other properties of the fluid but depends on the geometry of the medium, $\beta_{T}$ is the coefficient of thermal expansion, $\beta_{C}$ is the coefficient of concentration expansion, $T$ is the temperature of the fluid, $T_{W}$ is the temperature of the fluid at the surface, $T_{\infty}$ is the temperature of the fluid outside the boundary layer, $k$ is the thermal conductivity of the fluid, $C_{P}$ is the specific heat at constant pressure $P ; D_{M}$ is the chemical molecular diffusivity; $k_{c}$ is the chemical reaction constant. Here, $\rho$ is the density of the fluid; $g$ is the acceleration due to gravity. The applied magnetic field $B_{0}$ is a constant. $j, \mu$ and $\gamma$ are non-negative. Boundary conditions

$$
\begin{aligned}
& \text { At } y=0: u=u_{w}=b x, \quad v=0, \quad N=-m \frac{\partial u}{\partial y}, \quad T=T_{w}=T_{\infty}+A_{0}\left(\frac{x}{l}\right)^{2}, \quad C=C_{w}=C_{\infty}+A_{1}\left(\frac{x}{l}\right)^{2}, \\
& \text { ]As } \quad y \rightarrow \infty: u \rightarrow 0, \quad N \rightarrow 0, \quad T \rightarrow T_{\infty}, \quad C \rightarrow C_{\infty} .
\end{aligned}
$$

The thermal conductivity $k$ is assumed to vary linearly with temperature and it is of the form $k=k_{\infty}(1+\varepsilon \theta(\eta))$ where $\theta(\eta)=\left(T-T_{\infty}\right) /\left(T_{W}-T_{\infty}\right)$ and $\varepsilon=\left(k_{w}-k_{\infty}\right) / k_{\infty}$ which depends on the nature of the fluid and is a small parameter. $k_{w}$ is the thermal conductivity at the surface, $k_{\infty}$ is the thermal conductivity of the fluid far away from the surface. In general, $\varepsilon$ is constant; $\varepsilon>0$ for air and liquids such as water, while $\varepsilon<0$ for fluids such as lubrication oils.

Following the Rosseland approximation the radiative heat flux $q_{r}$ is modelled as $q_{r}=-\left(4 \sigma^{*} / 3 k^{*}\right)\left(\partial T^{4} / \partial y\right)$ where $\sigma^{*}$ is the Stefan- Boltzmann constant and $k^{*}$ is the mean absorption coefficient. $T^{4}$ can be expressed by using Taylor's series as $T^{4}=4 T_{\infty}^{3} T-3 T_{\infty}^{4}$.

$$
\begin{aligned}
& q_{r}=-\left(4 \sigma^{*} / 3 k^{*}\right) \frac{\partial}{\partial y}\left(4 T_{\infty}^{3} T-3 T_{\infty}^{4}\right)=-\left(16 \sigma^{*} / 3 k^{*}\right) T_{\infty}^{3} \frac{\partial T}{\partial y}, \\
& \frac{\partial q_{r}}{\partial y}=-\frac{16 \sigma^{*} T_{\infty}^{3}}{3 k^{*}} \frac{\partial^{2} T}{\partial y^{2}} .
\end{aligned}
$$

Using this in Eq.(2.4) we get

$$
u \frac{\partial T}{\partial x}+v \frac{\partial T}{\partial y}=\frac{1}{\rho C_{p}} \frac{\partial}{\partial y}\left(k \frac{\partial T}{\partial y}\right)-\frac{1}{\rho C_{p}} \frac{\partial}{\partial y}\left(-\left(16 \sigma^{*} / 3 k^{*}\right) T_{\infty}^{3}(\partial T / \partial y)\right)+\left(\frac{D_{M} K_{T}}{C_{S} C_{P}}\right) \frac{\partial^{2} C}{\partial y^{2}}
$$




$$
u \frac{\partial T}{\partial x}+v \frac{\partial T}{\partial y}=\frac{1}{\rho C_{p}} \frac{\partial}{\partial y}\left(\left(k+\frac{16 \sigma^{*} T_{\infty}^{3}}{3 k^{*}}\right) \frac{\partial T}{\partial y}\right)+\left(\frac{D_{M} K_{T}}{C_{S} C_{P}}\right) \frac{\partial^{2} C}{\partial y^{2}}
$$

To investigate the effect of different surface conditions, we choose a linear relationship between the microrotation variable $N$ and the surface stress $\frac{\partial u}{\partial y}$ in the boundary conditions (2.6); $m$ is the proportionality constant. Here, the microrotation parameter $m$ ranges between 0 and $l(0 \leq m \leq 1)$ when $m=0$, we have $N=0$, which is a generalisation of the no-slip condition, that is, the particle density is sufficiently large so that microelements close to the surface are not able to translate or rotate. The case $m=0.5$ represents the vanishing of the anti-symmetric part of the stress tensor and represents weak concentration of the microelements. For this case, in a fine particle suspension, the particle spin is equal to the fluid velocity at the surface. The value of $m=1$ is used for the modelling of a turbulent flow inside the boundary layers of microrotation.

Dimensional Analysis: We consider the following dimensionless variable

$$
\begin{aligned}
& \eta=y\left(\frac{b}{v}\right)^{\frac{l}{2}}, \quad N=b x\left(\frac{b}{v}\right)^{\frac{1}{2}} h(\eta), \quad u=b x f^{\prime}(\eta), \\
& v=-(b v)^{\frac{1}{2}} f(\eta), \quad \theta(\eta)=\frac{\left(T-T_{\infty}\right)}{\left(T_{w}-T_{\infty}\right)}, \quad \varphi(\eta)=\frac{\left(C-C_{\infty}\right)}{\left(C_{w}-C_{\infty}\right)} .
\end{aligned}
$$

Introducing these variables in Eqs (2.2), (2.3), (2.4) and (2.5), we get the following dimensionless forms of the equations

$$
\begin{aligned}
& \left(1+K_{m p}\right) f^{\prime \prime \prime}+f f^{\prime \prime}-f^{\prime 2}+K_{m p} h^{\prime}+\mathrm{Gr}_{\mathrm{T}} \theta+\mathrm{Gr}_{\mathrm{C}} \varphi-(M+(1 / \mathrm{Da})) f^{\prime}-\mathrm{Fs}\left(f^{\prime}\right)^{2}=0, \\
& \left(1+K_{m p} / 2\right) h^{\prime \prime}+\left(h^{\prime} f-h f^{\prime}\right)-K_{m p}\left(2 h+f^{\prime \prime}\right)=0, \\
& \frac{(1+\varepsilon \theta+R)}{\operatorname{Pr}} \theta^{\prime \prime}+f \theta^{\prime}-2 f^{\prime} \theta+\operatorname{Du} \varphi^{\prime \prime}+\frac{1}{\operatorname{Pr}} \varepsilon \theta^{\prime 2}=0, \\
& \frac{1}{\mathrm{Sc}} \varphi^{\prime \prime}+f \varphi^{\prime}-2 f^{\prime} \varphi-K_{c} \varphi+\operatorname{Sr} \theta^{\prime \prime}=0,
\end{aligned}
$$

where: $j=\frac{v x}{u_{w}}$ is the micro-inertia per unit mass, $\mathrm{M}=\frac{\sigma B_{0}^{2}}{\rho b}$ magnetic parameter (Hartmann number), $\mathrm{Gr}_{\mathrm{T}}=\frac{g \beta_{T}\left(T_{W}-T_{\infty}\right)}{b^{2}}$ thermal Grashof number, $\mathrm{Gr}_{\mathrm{c}}=\frac{g \beta_{C}\left(C_{W}-C_{\infty}\right)}{b^{2}}$ concentration Grashof number, $K_{m p}=\frac{k_{v}}{\mu}$ the micropolar or material parameter, $\gamma=\left(\mu+\frac{k_{v}}{2}\right), \frac{\gamma}{j \rho v}=1+\frac{K_{m p}}{2}, \frac{k_{v}}{j \rho b}=\frac{b k_{v}}{v \rho b}=\frac{k_{v}}{\mu}=K_{m p}$ 
the spin gradient viscosity, $j=\frac{v x}{u_{w}}=\frac{v}{b}=$ reference length, the micro-inertia per unit mass, $\mathrm{Da}=\frac{b k_{p}}{\mathrm{v}}$ the Darcy porosity parameter, $\mathrm{Fs}=\frac{C_{E} x}{\sqrt{k_{p}}}$ the Forchheimer parameter, $\operatorname{Pr}=\frac{\mathrm{v}}{\alpha}=\frac{\mathrm{v} \rho C_{p}}{k}$ the Prandtl number, $R=\frac{16 \sigma^{*} T_{\infty}^{3}}{3 k^{*} k}$ the radiation parameter, $\mathrm{Du}=\frac{D_{M} K_{T}\left(C_{W}-C_{\infty}\right)}{v C_{S} C_{P}\left(T_{W}-T_{\infty}\right)}=\frac{D_{M} K_{T} A_{I}}{v C_{S} C_{P} A_{0}}$ Dufour (thermo-diffusion) parameter, where $A_{0}=T_{W}-T_{\infty}, A_{l}=C_{W}-C_{\infty}, \quad \mathrm{Sc}=\frac{v}{D_{M}} \quad$ Schmidt number, $\quad \mathrm{Sr}=\frac{D_{M} K_{T}\left(T_{W}-T_{\infty}\right)}{\mathrm{v} T_{M}\left(C_{W}-C_{\infty}\right)}$ $=\frac{D_{M} K_{T} A_{0}}{v T_{M} A_{l}}$ Soret (diffusion-thermo) parameter; $K_{c}=\frac{k_{c}}{b}$ chemical reaction parameter; and the corresponding boundary conditions are as follows

$$
\begin{aligned}
& f(0)=0, \quad f^{\prime}(0)=1, \quad h(0)=-m f^{\prime \prime}(0) \quad \theta(0)=1 \quad, \varphi(0)=1, \\
& f^{\prime}(\infty)=0 \quad h(\infty)=0, \theta(\infty)=0, \varphi(\infty)=0
\end{aligned}
$$

The quantities of main physical interest are the skin friction coefficient (rate of shear stress), the couple stress coefficient at the surface, the Nusselt number (rate of heat transfer) and the Sherwood number (rate of mass transfer).

The local skin friction coefficient is defined as

$$
C_{f}=\frac{\tau_{W}}{\frac{1}{2} \rho u_{w}^{2}}=-2\left(\operatorname{Re}_{x}\right)^{-\frac{1}{2}} f^{\prime \prime}(0) .
$$

The couple stress coefficient at the surface is defined as follows

$$
C_{r}=\frac{\frac{\gamma}{k_{v}}\left(\frac{\partial N}{\partial y}\right)_{y=0}}{\gamma U_{0}^{3}\left(2 k_{v} v^{2}\right)}=\left(\operatorname{Re}_{x}\right)^{-1} h^{\prime}(0) .
$$

The rate of heat transfer in terms of the dimensionless Nusselt number is defined as follows

$$
\mathrm{Nu}_{x}=\frac{x q_{W}}{k\left(T_{W}-T_{\infty}\right)}=-2\left(\operatorname{Re}_{x}\right)^{-\frac{1}{2}} \theta^{\prime}(0)
$$

The rate of mass transfer in terms of the dimensionless Sherwood number is defined as follows

$$
\mathrm{Sh}_{x}=\frac{x J_{W}}{D_{M}\left(C_{W}-C_{\infty}\right)}=-2\left(\operatorname{Re}_{x}\right)^{-\frac{1}{2}} \varphi^{\prime}(0) .
$$

The equation defining the surface shear stress is defined as follows 


$$
\tau_{W}=\left(\mu+k_{v}\right)\left(\frac{\partial u}{\partial y}\right)_{y=0}+\left(k_{v} N\right)_{y=0}
$$

The heat flux is defined as follows

$$
q_{W}=-k\left(\frac{\partial T}{\partial y}\right)_{y=0} .
$$

The mass flux is defined as follows

$$
J_{W}=-D_{M}\left(\frac{\partial C}{\partial y}\right)_{y=0}
$$

The local Reynold number, $\operatorname{Re}_{x}=\frac{u_{w} x}{v}$.

\section{Method of numerical solution}

The numerical solutions are obtained using the above equations for some values of the governing parameters, namely, the magnetic parameter $(M)$, the Dufour number $(\mathrm{Du})$, the Soret number $(\mathrm{Sr})$, thermal coefficient parameter $(e)$ and slip parameter $(m)$. Effects of $M, \mathrm{Sr}$, and Du on the steady boundary layers in fluid flow region are discussed in detail. The numerical computation is done using the MATLAB in-built Numerical Solver bvp4c. In the computation we have taken $\eta_{\infty}=8.0$ and the axis according to the clear figure-visibility.

\section{Result and discussion}

The non-dimensional linear velocity $f^{\prime}(\eta)$, angular velocity $h(\eta)$, temperature $\theta(\eta)$, and concentration $\varphi(\eta)$ for various values of different parameters are shown in Figs 2 to 18.

Figure 2 shows that the velocity field decreases asymptotically with the increase in the magnetic parameter $M$. The fluid velocity decreases sharply when $0<\eta<2.5$ and when $2.5<\eta$, it decreases slowly and almost becomes constant. This shows that the velocity boundary layer thickness decreases with the increase in the magnetic parameter. Figure 3 shows that at the solid -fluid inter surface of the fluid, the fluid micro-rotation (angular velocity of fluid in fluid flow region) field increases asymptotically with the increase in the magnetic parameter $M$. The fluid angular velocity decreases sharply between $0<\eta<2.5$ and when $2.5<\eta$, it decreases slowly and almost becomes constant. For $M=0$ and 0.1 , micro rotation profiles have points of inflexion in the interval $\{2,3]$. This shows that the micro-rotation boundary layer thickness increases with the increase in the magnetic parameter. Figure 4 shows that the temperature field increases asymptotically with the increase in magnetic parameter $M$. The temperature decreases sharply when $0<\eta<4$ and when $4<\eta$, it decreases slowly and almost becomes constant. It shows that the thermal boundary layer thickness increases with the increase in the magnetic parameter $M$.

Figure 5 shows the concentration field increases asymptotically with the increase in magnetic parameter $\mathrm{M}$. The concentration decreases sharply when $0<\eta<2.5$ and when $2.5<\eta$, it decreases slowly and almost becomes constant. This shows concentration boundary layer thickness increases with the increase in the magnetic parameter $M$. 
Figure 6 shows that the velocity field increases asymptotically with the increase in Dufour parameter Du. The velocity decreases sharply when $0<\eta<2.5$ and when $2.5<\eta$, it decreases slowly and almost becomes constant. This shows that the velocity boundary layer thickness increases with the increase in the Dufour parameter Du. Figure 7 shows that at the solid -fluid inter surface of the fluid, the fluid microrotation (angular velocity of fluid in fluid flow region) field decreases asymptotically with the increase of the Dufour parameter Du. The fluid angular velocity decreases sharply between in $0<\eta<2.5$ and when $2.5<\eta$, it decreases slowly and almost becomes constant. For $0<\eta<2.5$ micro rotation profiles decrease with the increase in the Dufour parameter $\mathrm{Du}$ and for $2.5<\eta$ micro rotation profiles increase very slowly with the increase in the Dufour parameter $\mathrm{Du}$. This shows that the micro-rotation boundary layer thickness decreases with the increase in the Dufour parameter Du. Figure 8 shows that the fluid temperature field increases asymptotically with the increase in the Dufour parameter Du. The temperature decreases sharply when $0<\eta<4$ and when $4<\eta$, it decreases slowly and almost becomes constant. This shows that the thermal boundary layer thickness increases with the increase in the Dufour parameter $\mathrm{Du}$.

Figure 9 shows that the fluid concentration field decreases asymptotically with the increase in the Dufour parameter. The concentration decreases sharply when $0<\eta<2.5$ and when $2.5<\eta$, it decreases slowly and almost becomes constant. This shows concentration boundary layer thickness decreases with the increase in the Dufour parameter. Figure 10 shows that the fluid velocity field decreases asymptotically with the increase in the Soret parameter. The velocity decreases sharply when $0<\eta<2.5$ and when $2.5<\eta$, it decreases slowly and almost becomes constant. This shows that the velocity boundary layer thickness decreases with the increase in the Soret parameter. Figure 11 shows that at the solid -fluid inter surface of the fluid, the fluid micro-rotation (angular velocity of fluid in fluid flow region) field increases asymptotically with the increase in the Soret parameter. The fluid angular velocity decreases sharply between in $0<\eta<2.5$ and when $2.5<\eta$, it decreases slowly and almost becomes constant. For $0<\eta<2.5$ micro rotation profiles increase with the increase in the Soret parameter and for $2.5<\eta$ micro rotation profiles decrease very slowly with the increase in the Soret parameter. This shows that the micro-rotation boundary layer thickness increases with the increase in the Soret parameter. Figure 12 shows that the fluid temperature field decreases asymptotically with the increase in the Soret parameter. The temperature decreases sharply when $0<\eta<4$ and when $4<\eta$, it decreases slowly and almost becomes constant. This shows that thermal boundary layer thickness decreases with the increase in the Soret parameter. Figure 13 shows that the fluid concentration field decreases asymptotically with the increase in the Soret parameter. The concentration decreases sharply when $0<\eta<2.5$ and when $2.5<\eta$, it decreases slowly and almost becomes constant. This shows that the concentration boundary layer thickness decreases with the increase in the Soret parameter. Figure 14 shows that the fluid velocity field increases asymptotically with the increase in the thermal coefficient (e). The velocity decreases sharply when $0<\eta<2.5$ and when $2.5<\eta$, it decreases slowly and almost becomes constant. This shows that velocity boundary layer thickness increases with the increase in the thermal coefficient.

Figure 15 shows that the solid -fluid surface of the fluid, the fluid micro-rotation (angular velocity of fluid in fluid flow region) field decreases asymptotically with the increase the thermal coefficient. The fluid angular velocity decreases sharply between in $0<\eta<2.5$ and when $2.5<\eta$, it decreases slowly and almost becomes constant. For $0<\eta<2.5$ micro rotation profiles decrease with the increase in the thermal coefficient and for $2.5<\eta$ micro rotation profiles increase very slowly with the increase in the thermal coefficient. This shows the micro-rotation boundary layer thickness decreases with the increase in the thermal coefficient.

Figure 16 shows that the fluid temperature field increases asymptotically with the increase in the thermal coefficient (e). The temperature decreases sharply when $0<\eta<4$ and when $4<\eta$, it decreases slowly and almost becomes constant. This shows that thermal boundary layer thickness increases with the increase in the thermal coefficient. Figure 17 shows that the fluid concentration field increases 
asymptotically with the increase in the thermal coefficient. The concentration decreases sharply when $0<\eta<2.5$ and when $2.5<\eta$, it decreases slowly and almost becomes constant. This shows that the concentration boundary layer thickness increases with the increase in the thermal coefficient. Figure 18 shows that at the solid -fluid surface of the fluid, the fluid micro-rotation (angular velocity of fluid in fluid flow region) field increases asymptotically with the increase in the velocity slip ' $m$ '. The fluid angular velocity decreases sharply in between $0<\eta<2.5$ and when $2.5<\eta$, it decreases slowly and almost becomes constant. For $M=0$ and 0.1 , micro rotation profiles have points of inflexion in the interval $(2,3]$. This shows that the micro-rotation boundary layer thickness increases with the increase in the velocity slip $(m)$.

To ensure the numerical accuracy, the values of $-\theta^{\prime}(0)$ by present method are compared with the results of Ishak et al. (2008), Chen (1998), Grubka and Bobba (1985) and Pal and Chatterjee (2015) in Tab. 1 for various values of $\mathrm{Pr}$ and $\mathrm{Da}=\infty, \mathrm{Fs}=0.0, \mathrm{Gr}_{\mathrm{T}}=0.0, \mathrm{Gr}_{\mathrm{c}}=0.0, K_{m p}=0.0, \mathrm{Sc}=0.0, m=0.0, M=0.0$, $\mathrm{Pr}=1.0, R=0.0, e=0.00, \mathrm{Du}=0.00, \mathrm{Sr}=0.00, K c=0.0$ and $f^{\prime \prime}(0)=0$ and those are found in excellent agreement with the present values. Thus, we are very much confident that the present results are accurate.

Table1. Comparison of the local Nusselt number $-\theta^{\prime}(0)$ with Ishak et al. (2008), Chen (1998), Grubka and Bobba (1985) and Pal and Chatterjee (2015), and present results for various values of $\mathrm{Pr}$ and $\mathrm{Da}=\infty, \mathrm{Fs}=0.0, \mathrm{Gr}_{\mathrm{T}}=0.0, \mathrm{Gr}_{\mathrm{c}}=0.0, K_{m p}=0.0, \mathrm{Sc}=0.0, m=0.0, M=0.0, \mathrm{Pr}=1.0, R=0.0, e=0.0, \mathrm{Du}=0.0$, $\mathrm{Sr}=0.0, K_{c}=0.0, f^{\prime}(0)=0.0$.

\begin{tabular}{|c|c|c|c|c|c|}
\hline \multirow[b]{2}{*}{$\operatorname{Pr}$} & \multicolumn{4}{|c|}{$\theta^{\prime}(0)$} & \multirow{2}{*}{$\begin{array}{l}\theta^{\prime}(0) \\
\text { Present } \\
\text { Results }\end{array}$} \\
\hline & $\begin{array}{l}\text { Ishak et al. } \\
\quad(2008)\end{array}$ & $\begin{array}{l}\text { Chen } \\
(1998)\end{array}$ & $\begin{array}{c}\text { Grubka and } \\
\text { Bobba } \\
(1985)\end{array}$ & $\begin{array}{c}\text { Pal and Chatterjee } \\
\text { (2015) }\end{array}$ & \\
\hline 1 & 1.3333 & 1.33334 & 1.3333 & 1.333333 & 1.333346 \\
\hline 3 & 2.5097 & 2.50997 & 2.5097 & 2.509725 & 2.509682 \\
\hline 10 & 4.7969 & 4.79686 & 4.7969 & 4.796873 & 4.796226 \\
\hline
\end{tabular}

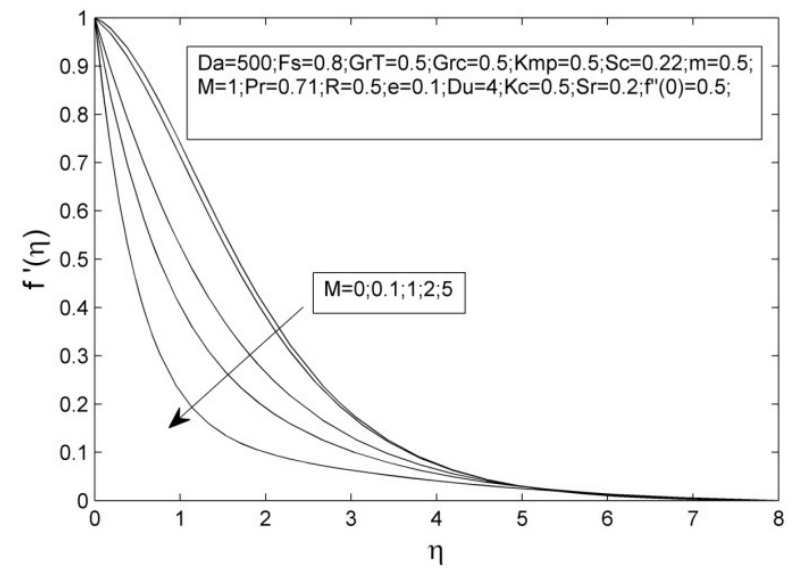

Fig.2. Variation in the value of fluid velocity with $M$, the magnetic parameter.

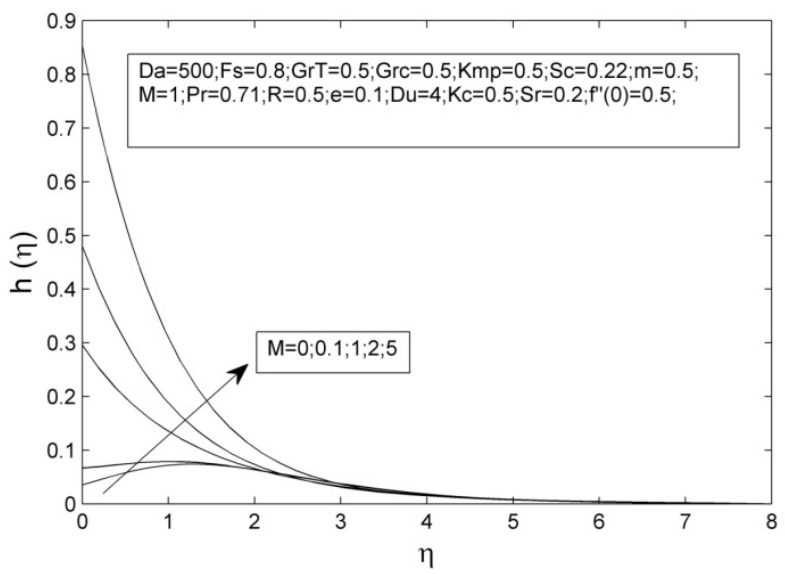

Fig.3. Variation in the value of angular velocity with $M$, the magnetic parameter. 


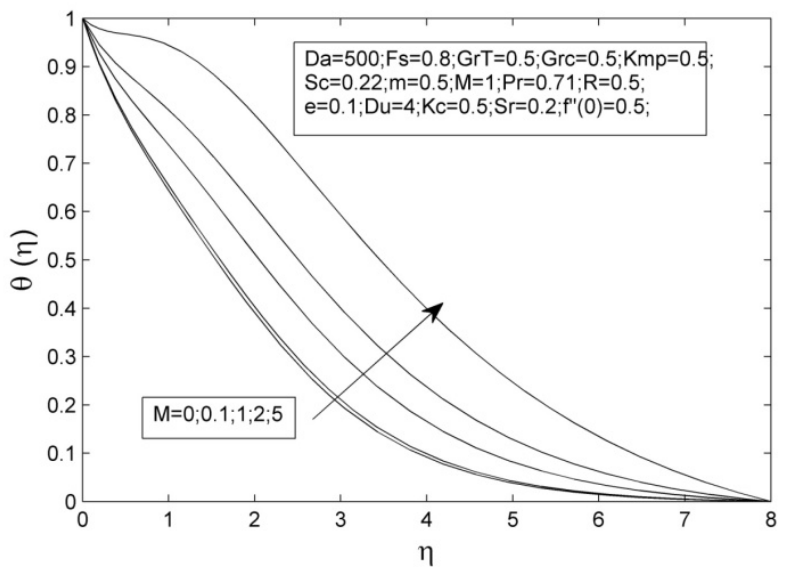

Fig.4. Variation in the value of temperature with $M$, Fig.5. Variation in the value of concentration with the magnetic parameter.

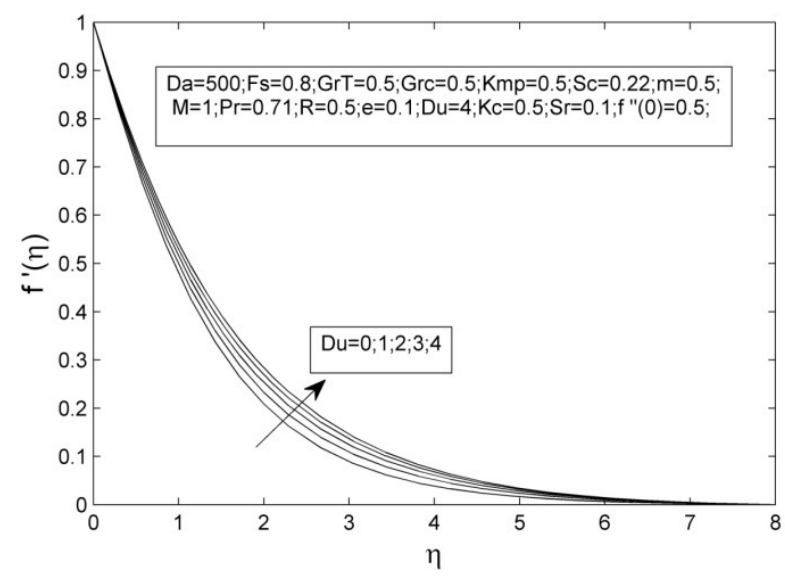

Fig.6. Variation in the value of fluid velocity with $\mathrm{Du}$, the Dufour parameter.

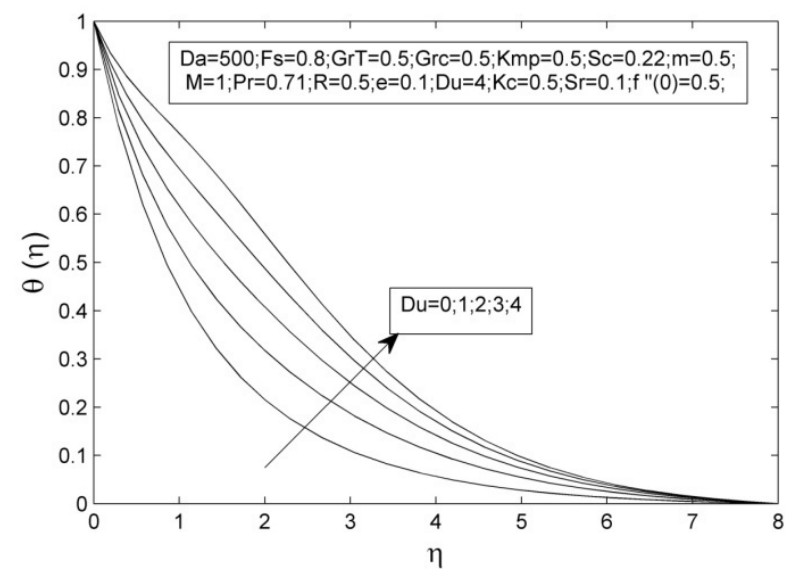

Fig.8. Variation in the value of temperature with $\mathrm{Du}$, the Dufour parameter.

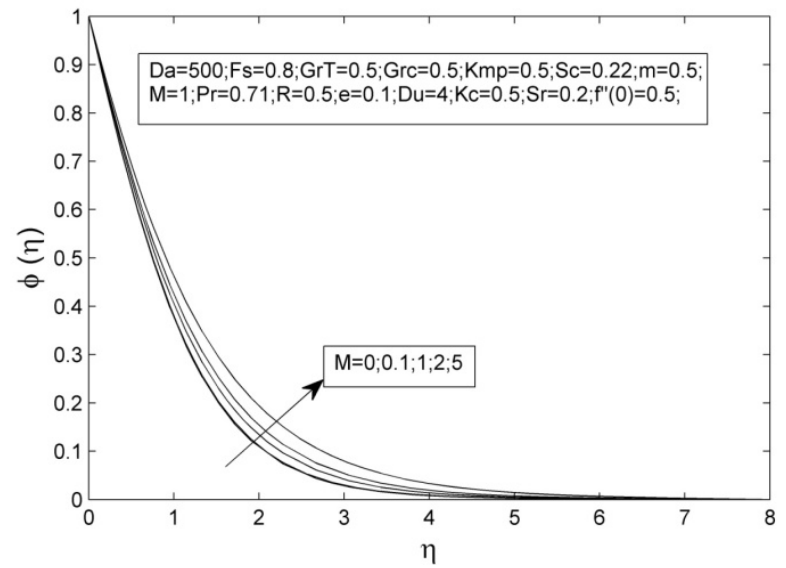
$M$, the magnetic parameter.

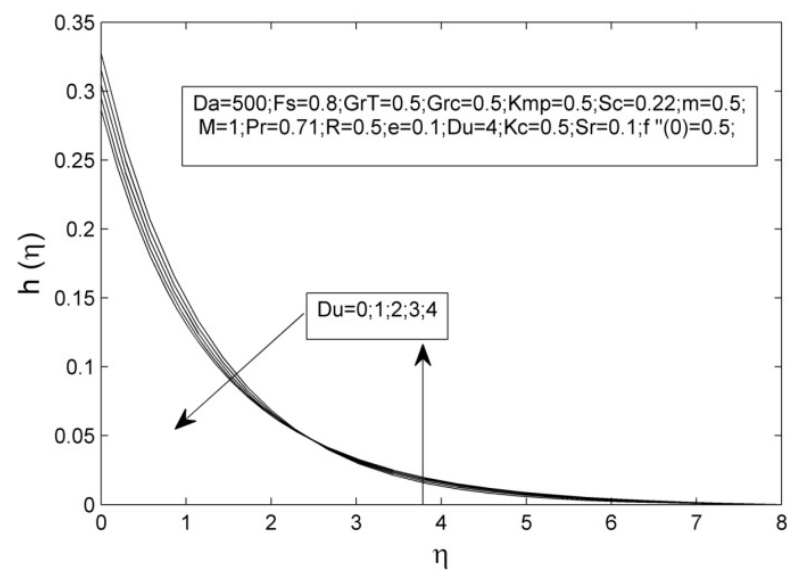

Fig.7. Variation in the value of angular velocity with $\mathrm{Du}$, the Dufour parameter.

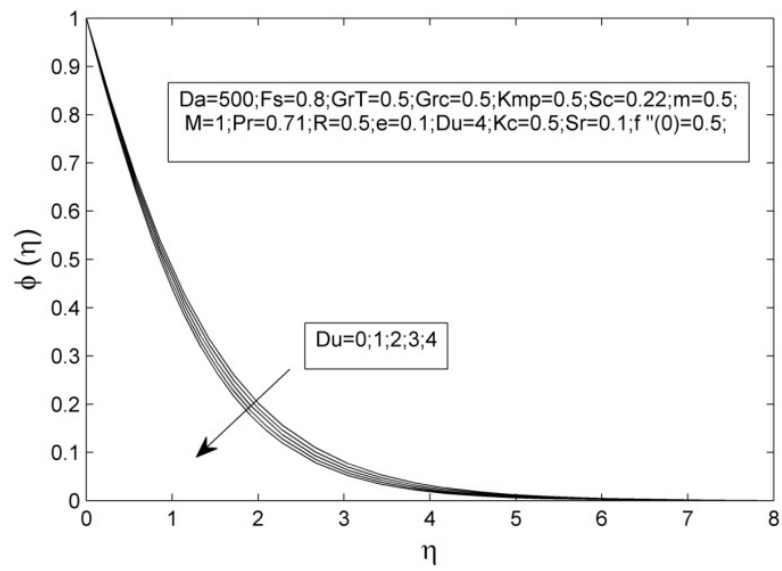

Fig.9. Variation in the value of concentration with $\mathrm{Du}$, the Dufour parameter. 


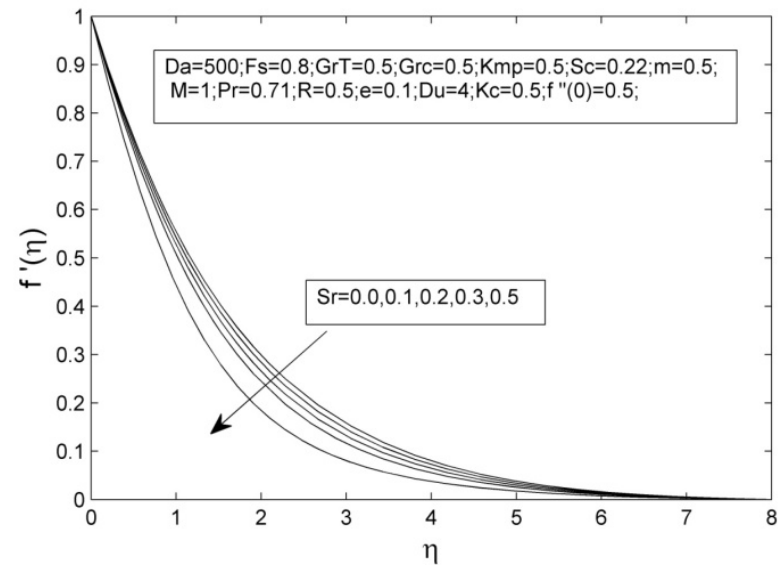

Fig.10. Variation in the value of fluid velocity with the Soret parameter.

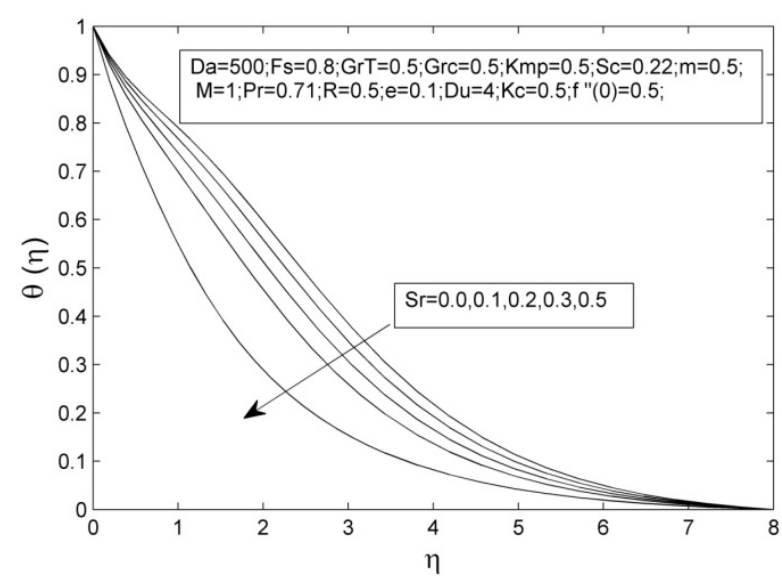

Fig.12. Variation in the value of temperature with the Soret parameter.

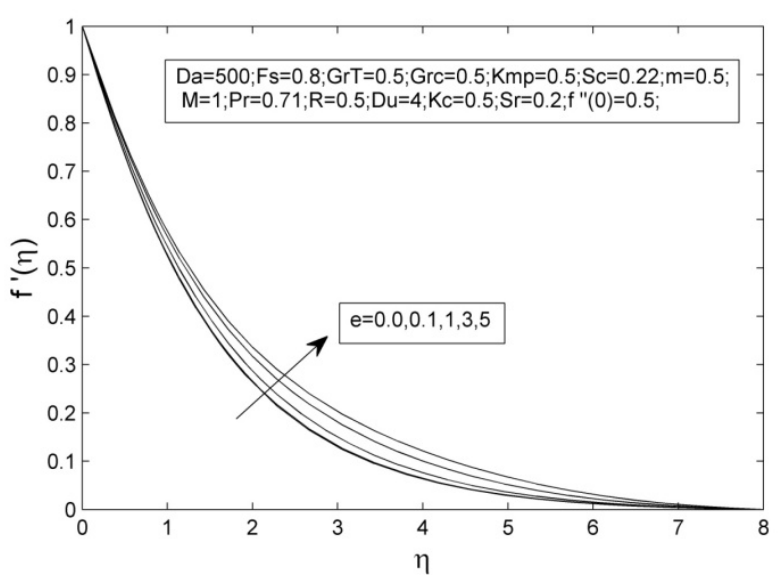

Fig.14. Variation in the value of fluid velocity with $e$, the thermal coefficient.

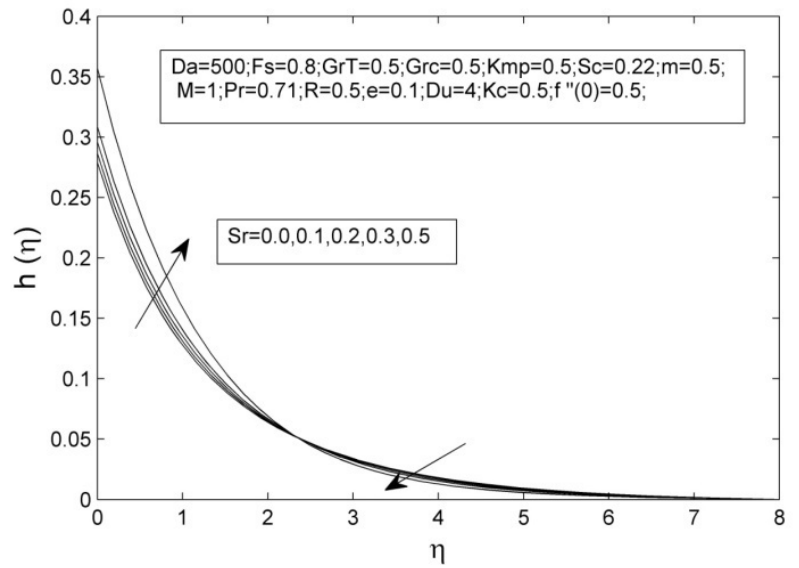

Fig.11. Variation in the value of angular velocity with the Soret parameter.

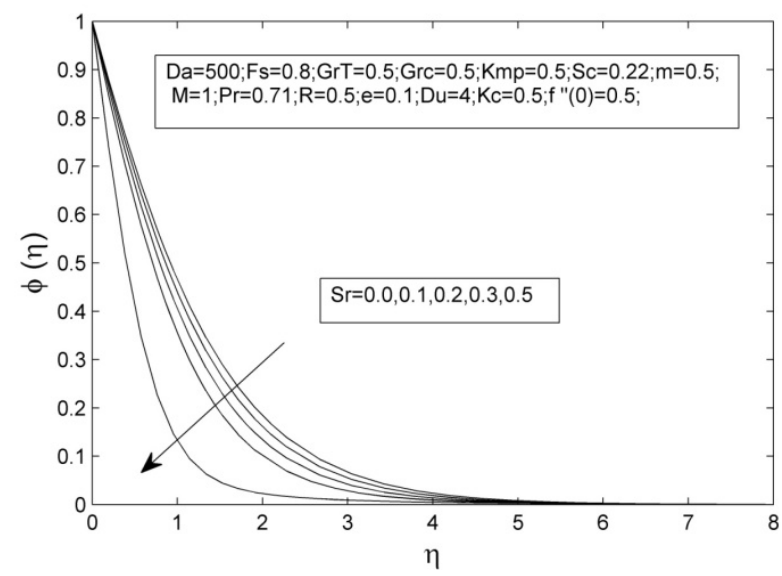

Fig.13. Variation in concentration with the Soret parameter $\mathrm{Sr}$.

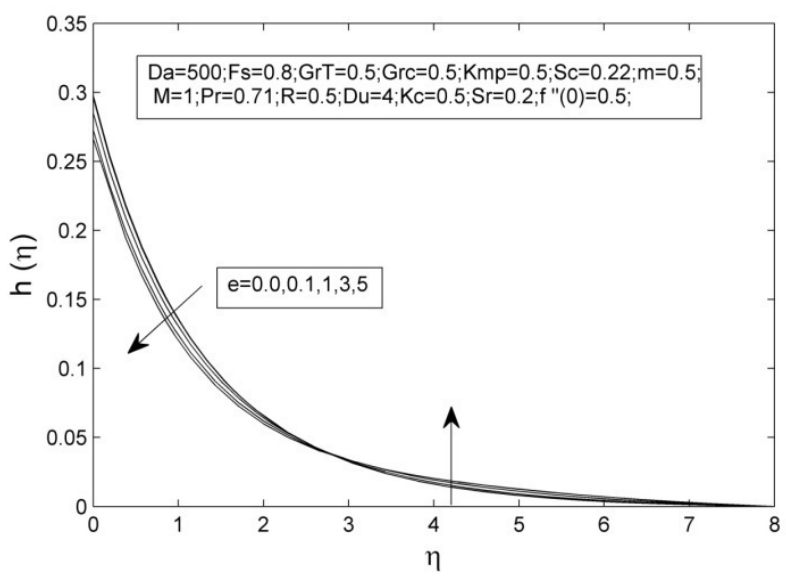

Fig.15. Variation in the value of angular velocity with $e$, the thermal coefficient. 


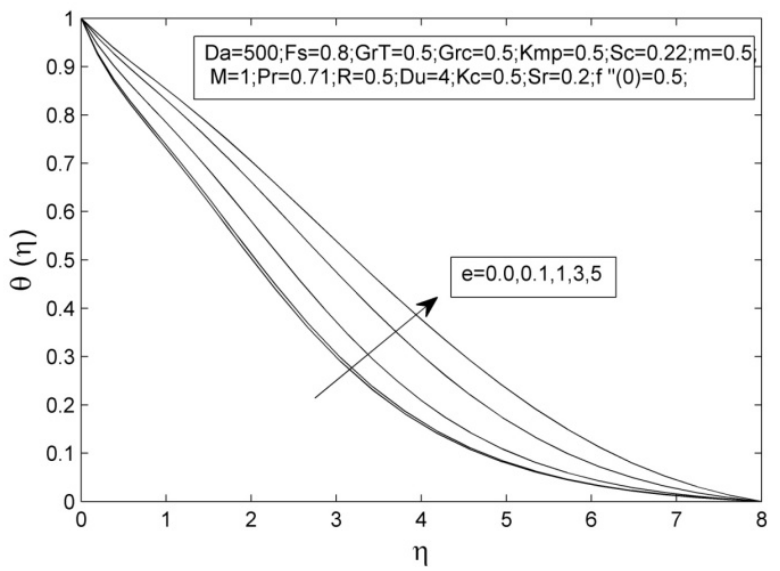

Fig.16. Variation in the temperature with $e$, the thermal coefficient.

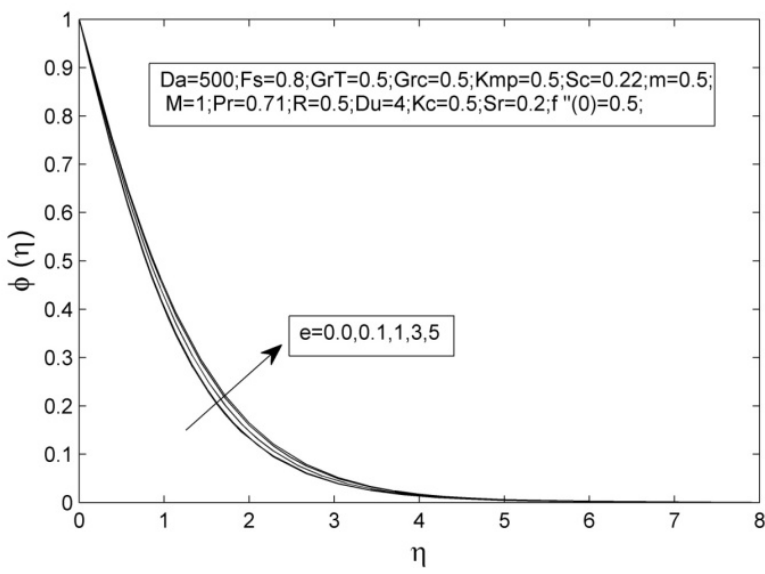

Fig.17. Variation of concentration with $e$, the thermal coefficient.

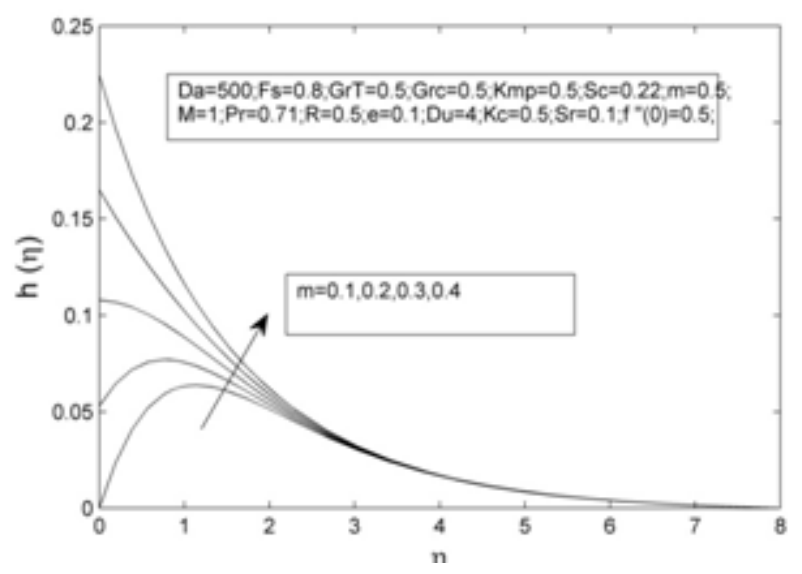

Fig.18. Variation in the angular velocity with the slip parameter.

Table 2. The values of the skin friction $\mathrm{C}_{\mathrm{f}}\left(f^{\prime \prime}(0)\right)$, dimensionless surface couple stress $\mathrm{C}_{\mathrm{r}}\left(h^{\prime}(0)\right)$, Nusselt number $\mathrm{Nu}\left(-\theta^{\prime}(0)\right)$ and Sherwood number $\mathrm{Sh}\left(-\varphi^{\prime}(0)\right)$ for different values of $\mathrm{Da}$ and $\mathrm{Fs}_{\mathrm{s}}=0.8$, $\mathrm{Gr}_{\mathrm{T}}=0.6, \mathrm{Gr}_{\mathrm{c}}=0.5, \mathrm{Kmp}=0.5, \mathrm{Sc}=0.22, M=1, \mathrm{Pr}=0.71, R=0.5, e=0.1, \mathrm{Du}=4, K c=0.5, \mathrm{Sr}=0.1$, $f^{\prime \prime}(0)=0.5, m=0.5$.

\begin{tabular}{|c|l|l|l|l|}
\hline $\mathrm{Da}$ & $f^{\prime \prime}(0)$ & $h^{\prime}(0)$ & $-\theta^{\prime}(0)$ & $-\phi^{\prime}(0)$ \\
\hline 100 & -0.5232968998 & -0.2130987767 & 0.4148029270 & 0.6842387169 \\
\hline 300 & -0.5203648452 & -0.2114961126 & 0.4155003921 & 0.6844328942 \\
\hline 500 & -0.5197776341 & -0.2111750671 & 0.4156400406 & 0.6844717840 \\
\hline
\end{tabular}

Table 2 shows that the effects of the Darcy parameter $(\mathrm{Da})$ on the skin friction $(C f)$, dimensionless wall couple stress $(\mathrm{Cr})$, Nusselt number and Sherwood number. It shows that the skin friction, dimensionless wall couple stress, Nusselt number and Sherwood number increase with the increase in the value of the Darcy parameter (Da). 
Table 3. The values of the skin friction $C_{f}\left(f^{\prime \prime}(0)\right)$, dimensionless surface couple stress $C_{r}\left(h^{\prime}(0)\right)$, Nusselt number $\mathrm{Nu}\left(-\theta^{\prime}(0)\right)$ and Sherwood number $\mathrm{Sh}\left(-\varphi^{\prime}(0)\right)$ for different values of Fs and $\mathrm{Da}=500$, $\mathrm{Fs}=0.8, \mathrm{Gr}_{\mathrm{T}}=0.5, \mathrm{Gr}_{\mathrm{c}}=0.5, \mathrm{Kmp}=0.5, \mathrm{Sc}=0.22, M=1, \mathrm{Pr}=0.71, R=0.5, e=0.1, \mathrm{Du}=4, K c=0.5$, $\mathrm{Sr}=0.1, f^{\prime \prime}(0)=0.5, m=0.5$.

\begin{tabular}{|l|l|l|l|l|}
\hline Fs & $f^{\prime \prime}(0)$ & $h^{\prime}(0)$ & $-\theta^{\prime}(0)$ & $-\phi^{\prime}(0)$ \\
\hline 0.4 & -0.6923363518 & -0.3067500491 & 0.3800429068 & 0.6741087526 \\
\hline 0.6 & -0.6343386592 & -0.2738148989 & 0.3890995886 & 0.6771447600 \\
\hline 0.8 & -0.5728824078 & -0.2387131577 & 0.3987757166 & 0.6803955008 \\
\hline
\end{tabular}

Table 3 shows the effects of the Forchheimer parameter (Fs) on the skin friction, dimensionless wall couple stress, Nusselt number and Sherwood number. It shows that the skin friction, dimensionless wall couple stress, Nusselt number and Sherwood number increases with the increase in the value of Forchheimer parameter (Fs).

Table 4. The values of the skin friction $C f,\left(f^{\prime \prime}(0)\right)$, dimensionless wall couple stress $C r,\left(h^{\prime}(0)\right)$, Nusselt number $\mathrm{Nu},\left(-\theta^{\prime}(0)\right.$ )and Sherwood number $\mathrm{Sh},\left(-\phi^{\prime}(0)\right)$ for different values of $\mathrm{Gr}_{\mathrm{T}}$ and $a=500$; $\mathrm{Fs}=0.8 ; \mathrm{Gr}_{\mathrm{T}}=0.5 ; \mathrm{Gr}_{\mathrm{C}}=0.5 ; \mathrm{Kmp}=0.5 ; \mathrm{Sc}=0.22 ; M=1 ; \mathrm{Pr}=0.71 ; R=0.5 ; \quad e=0.1 ; \mathrm{Du}=4 ; K c=0.5$; $\mathrm{Sr}=0.1 ; f^{\prime \prime}(0)=0.5 ; m=0.5$.

\begin{tabular}{|c|c|c|c|c|}
\hline GrT & $f^{\prime \prime}(0)$ & $h^{\prime}(0)$ & $-\theta^{\prime}(0)$ & $-\phi^{\prime}(0)$ \\
\hline 0.4 & -0.7419638023 & -0.3220532467 & 0.3319502829 & 0.666202065 \\
\hline 0.6 & -0.6273918455 & -0.2663504570 & 0.3797860892 & 0.6760412478 \\
\hline 0.8 & -0.5197776341 & -0.2111750671 & 0.4156400406 & 0.6844717840 \\
\hline
\end{tabular}

Table 4 shows that the effects of the thermal Grashof parameter $\left(\mathrm{Gr}_{\mathrm{T}}\right)$ on the skin friction, dimensionless wall couple stress, Nusselt number and Sherwood number. It shows that the skin friction, dimensionless wall couple stres, Nusselt number and Sherwood number increase with the increase in the value of the Grashof temperature parameter.

Table 5. Shows that the various values of the skin friction $C f,\left(f^{\prime \prime}(0)\right)$, dimensionless wall couple stress, ( $\left.h^{\prime}(0)\right)$, Nusselt number, $\left(-\theta^{\prime}(0)\right)$ and Sherwood number Sh, $\left(-\phi^{\prime}(0)\right)$ for different values of $\mathrm{Gr}_{\mathrm{C}}$ and $\mathrm{Da}=500 ; \mathrm{Fs}=0.8 ; \mathrm{Gr}_{\mathrm{T}}=0.5 ; \mathrm{Gr}_{\mathrm{c}}=0.5 ; \mathrm{Kmp}=0.5 ; \mathrm{Sc}=0.22 ; M=1 ; \mathrm{Pr}=0.71 ; R=0.5 ; e=0.1 ; \mathrm{Du}=4$; $K c=0.5 ; \mathrm{Sr}=0.1 ; f^{\prime \prime}(0)=0.5 ; m=0.5$.

\begin{tabular}{|c|c|c|c|c|}
\hline Grc & $f^{\prime \prime}(0)$ & $h^{\prime}(0)$ & $-\theta^{\prime}(0)$ & $-\phi^{\prime}(0)$ \\
\hline 0.4 & -0.6891462664 & -0.3027219793 & 0.3752254971 & 0.6732310281 \\
\hline 0.6 & -0.6114477561 & -0.2601329838 & 0.3911533661 & 0.6780545407 \\
\hline 0.8 & -0.5344995269 & -0.2172142340 & 0.4061880046 & 0.6826918568 \\
\hline
\end{tabular}

Table 5 shows that the effects of the concentration Grashof parameter $\left(\mathrm{Gr}_{\mathrm{C}}\right)$ on the skin friction $(C f)$, dimensionless wall couple stress $\mathrm{Cr}$, Nusselt number $\mathrm{Nu}$ and Sherwood number Sh. It shows that the skin friction, dimensionless wall couple stress, Nusselt number and Sherwood number increase with the increase in the value of the Grashof concentration parameter. 
Table 6. The values of the skin friction $C f,\left(f^{\prime \prime}(0)\right)$, dimensionless wall couple stress $C r,\left(h^{\prime}(0)\right)$, Nusselt number $\mathrm{Nu},\left(-\theta^{\prime}(0)\right)$ and Sherwood number Sh, $\left(-\phi^{\prime}(0)\right)$ for different values of Kmp and $\mathrm{Da}=500 ; \mathrm{Fs}=0.8 ; \mathrm{Gr}_{\mathrm{T}}=0.6 ; \mathrm{Gr}_{\mathrm{c}}=0.5 ; \mathrm{Kmp}=0.5 ; \mathrm{Sc}=0.22 ; M=1 ; \operatorname{Pr}=0.71 ; R=0.5 ; e=0.1 ; \mathrm{Du}=4 ;$ $K c=0.5 ; \mathrm{Sr}=0.1 ; f^{\prime \prime}(0)=0.5 ; m=0.5$.

\begin{tabular}{|c|c|c|c|c|}
\hline $\mathrm{Kmp}$ & $f^{\prime \prime}(0)$ & $h^{\prime}(0)$ & $-\theta^{\prime}(0)$ & $-\phi^{\prime}(0)$ \\
\hline 0.4 & -0.5238358848 & -0.2227831673 & 0.4137682343 & 0.6840377088 \\
\hline 0.6 & -0.5156431164 & -0.2009795469 & 0.4174565801 & 0.6848951515 \\
\hline 0.8 & -0.5073869212 & -0.1837565643 & 0.4209411691 & 0.6857081329 \\
\hline
\end{tabular}

Table 6 shows that the effects of the micropolar parameter, $K_{n p}$ on the skin friction, dimensionless wall couple stress $C r$, Nusselt number and Sherwood number. It shows that the skin friction $C f$, dimensionless wall couple stress $\mathrm{Cr}$, Nusselt number $\mathrm{Nu}$ and Sherwood number increase with the increase in the value of micropolar parameter.

Table 7. The values of the skin friction $C f,\left(f^{\prime \prime}(0)\right)$, dimensionless wall couple stress $C r,\left(h^{\prime}(0)\right)$, Nusselt number $\mathrm{Nu},\left(-\theta^{\prime}(0)\right)$ and Sherwood number $\mathrm{Sh},\left(-\phi^{\prime}(0)\right)$ for different values of $\mathrm{Pr}$ and $\mathrm{Da}=500$; $\mathrm{Fs}=0.8 ; \mathrm{Gr}_{\mathrm{T}}=0.5 ; \mathrm{Ge}_{\mathrm{c}}=0.5 ; \mathrm{Kmp}=0.5 ; \mathrm{Sc}=0.22 ; M=1 ; m=0.5 ; R=0.5 ; \quad e=0.1 ; \mathrm{Du}=4 ; K c=0.5$; $\mathrm{Sr}=0.1 ; f^{\prime \prime}(0)=0.5$.

\begin{tabular}{|c|c|c|c|c|}
\hline $\operatorname{Pr}$ & $f^{\prime \prime}(0)$ & $h^{\prime}(0)$ & $-\theta^{\prime}(0)$ & $-\phi^{\prime}(0)$ \\
\hline 1 & -0.5877606189 & -0.2441906200 & 0.4890258990 & 0.6995598113 \\
\hline 2 & -0.6407913311 & -0.2648382955 & 0.8040876448 & 0.8002199228 \\
\hline 3 & -0.7216655338 & -0.2978096753 & 1.1908847741 & 1.0429179103 \\
\hline
\end{tabular}

Table 7 shows that the effects of the Prandtl parameter on the skin friction $(C f)$, dimensionless wall couple stress $C r$, Nusselt number and Sherwood number. It shows that the skin friction $C f$, dimensionless wall couple stress Cr decrease, Nusselt number and Sherwood number increase with the increase in the value of the Prandtl number.

Table 8. The values of the skin friction $C f,\left(f^{\prime \prime}(0)\right)$, dimensionless wall couple stress $C r,\left(h^{\prime}(0)\right)$, Nusselt number $\mathrm{Nu},\left(-\theta^{\prime}(0)\right)$ nand Sherwood number $\mathrm{Sh},\left(-\phi^{\prime}(0)\right)$ for different values of $R$ and $\mathrm{Da}=500$; $\mathrm{Fs}=0.8 ; \mathrm{Gr}_{\mathrm{T}}=0.5 ; \mathrm{Gr}_{\mathrm{C}}=0.5 ; \mathrm{Kmp}=0.5 ; \mathrm{Sc}=0.22 ; M=1 ; \mathrm{Pr}=0.71 ; R=0.5 ; e=0.1 ; \mathrm{Du}=4 ; K c=0.5 ;$ $\mathrm{Sr}=0.1 ; f^{\prime \prime}(0)=0.5 ; m=0.5$.

\begin{tabular}{|c|c|c|c|c|}
\hline $\mathrm{R}$ & $f^{\prime \prime}(0)$ & $h^{\prime}(0)$ & $-\theta^{\prime}(0)$ & $-\phi^{\prime}(0)$ \\
\hline 0.2 & -0.5815547952 & -0.2418564517 & 0.4495261450 & 0.6910481957 \\
\hline 0.4 & -0.5754249947 & -0.2396276727 & 0.4135433236 & 0.6833552017 \\
\hline 0.6 & -0.5706029556 & -0.2378983543 & 0.3856487998 & 0.6778604185 \\
\hline
\end{tabular}

Table 8 shows that the effects of the radiation parameter on the skin friction, dimensionless wall couple stress, Nusselt number and Sherwood number. It shows that the skin friction, dimensionless wall couple stress $\mathrm{Cr}$ increase, Nusselt number and Sherwood number decrease with the increase in the value of the radiation parameter. 
Table 9. The values of the skin friction $C f,\left(f^{\prime \prime}(0)\right)$, dimensionless wall couple stress $C r,\left(h^{\prime}(0)\right)$, Nusselt number $\mathrm{Nu},\left(-\theta^{\prime}(0)\right)$ and Sherwood number Sh, $\left(-\phi^{\prime}(0)\right)$ for different values of Sc and $\mathrm{Da}=500$; $\mathrm{Fs}=0.8 ; \mathrm{Gr}_{\mathrm{T}}=0.5 ; \mathrm{Gr}_{\mathrm{c}}=0.5 ; \mathrm{Kmp}=0.5 ; \mathrm{Sc}=0.22 ; M=1 ; \mathrm{Pr}=0.71 ; R=0.5 ; e=0.1 ; \mathrm{Du}=4 ; K c=0.5$; $\mathrm{Sr}=0.1 ; f^{\prime \prime}(0)=0.5 ; m=0.5$.

\begin{tabular}{|c|c|c|c|c|}
\hline $\mathrm{Sc}$ & $f^{\prime \prime}(0)$ & $h^{\prime}(0)$ & $-\theta^{\prime}(0)$ & $-\phi^{\prime}(0)$ \\
\hline 0.2 & -0.5721485289 & -0.238587915 & 0.429468888 & 0.653650121 \\
\hline 0.4 & -0.57 & -0.2 & 0.15 & 0.87578 \\
\hline 0.6 & -0.5730513358 & -0.2367478821 & -0.0840118311 & 1.0403330825 \\
\hline
\end{tabular}

Table 9 shows that the effects of the Schmidt parameter on the skin friction, dimensionless wall couple stress, the Nusselt number and Sherwood number. It shows the skin friction $C f$, first decreases and then increases, the dimensionless wall couple stress $\mathrm{Cr}$ increases, the Nusselt number decrease and Sherwood number increases with the increase in the value of the Schmidt parameter.

Table 10. The values of the skin friction $C f,\left(f^{\prime \prime}(0)\right)$, the dimensionless wall couple stress $C r,\left(h^{\prime}(0)\right)$, the Nusselt number $\mathrm{Nu},\left(-\theta^{\prime}(0)\right)$ and Sherwood number Sh, $\left(-\phi^{\prime}(0)\right)$ for different values of $K c$ and

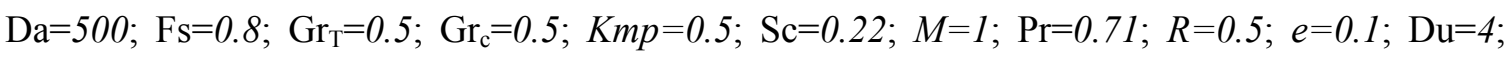
$K c=0.5 ; \mathrm{Sr}=0.1 ; f^{\prime \prime}(0)=0.5 ; m=0.5$.

\begin{tabular}{|c|c|c|c|c|}
\hline $\mathrm{Kc}$ & $f^{\prime \prime}(0)$ & $h^{\prime}(0)$ & $-\theta^{\prime}(0)$ & $-\phi^{\prime}(0)$ \\
\hline 0.2 & -0.5717526148 & -0.2384718973 & 0.4621701275 & 0.62790533 \\
\hline 0.4 & -0.5 & -0.2 & 0.41 & 0.66323 \\
\hline 0.6 & -0.5732270304 & -0.2387851687 & 0.3782987599 & 0.6972477499 \\
\hline
\end{tabular}

Table 10 shows that the effects of the chemical reaction parameter on the skin friction $C f$, dimensionless wall couple stress $\mathrm{Cr}$, Nusselt number $\mathrm{Nu}$, and Sherwood number $\mathrm{Sh}$. It shows that the skin friction $C f$, dimensionless wall couple stress $\mathrm{Cr}$, Nusselt number $\mathrm{Nu}$ decrease and the Sherwood number increase with the increase in the value of the chemical reaction parameter.

\section{Conclusions}

- With the increase in the value of $M$, the velocity boundary layer thickness decreases, whereas the microrotation boundary layer thickness, the thermal boundary layer thickness and the concentration boundary layer thickness increase.

- With the increase in the value of $\mathrm{Du}$, the velocity boundary layer thickness increases, the microrotation boundary layer thickness decreases, the thermal boundary layer thickness increases, the concentration boundary layer thickness decreases.

- With the increase in the value of $\mathrm{Sr}$, the velocity boundary layer thickness decreases, the microrotation boundary layer thickness increases, the thermal boundary layer thickness decreases, concentration boundary layer thickness decreases

- The skin friction $C f$, the dimensionless wall couple stress $C r$, Nusselt number and Sherwood number increase with the increase in the value of Darcy, the Forchheimer number, Grashof number, modified Grashof number concentration and micropolar parameters .

- As the value of the Prandtl parameter increases the skin friction $C_{f}$ dimensionless wall couple stress $\mathrm{Cr}$, decrease and Nusselt number $\mathrm{Nu}$, and Sherwood number Sh, increase. 
- As the value of radiation parameter increases the skin friction $C f$, dimensionless wall couple stress $\mathrm{Cr}$ increase and Nusselt number and Sherwood number decrease.

- As the value of the Schmidt parameter increases the skin friction first decreases and then increases, dimensionless wall couple stress $\mathrm{Cr}$ and the Sherwood number increase, the Nusselt number decreases.

- As the value of the chemical reaction parameter increases, the skin friction, dimensionless wall couple stress $\mathrm{Cr}$ and the Nusselt number decrease and the Sherwood number increases.

\section{Acknowledgement}

I am thankful to the Department of Mathematics, K.L. University, for its heartly support in our research work, and for providing us research facilities.

\section{Nomenclature}

$B_{0} \quad$ - applied magnetic field

$C_{E} \quad$ - drag coefficient which - independent of viscosity

$C_{P} \quad$ - specific heat at constant pressure

$D_{M} \quad$ - chemical molecular diffusivity

$g$ - acceleration due to gravity

$j$ - micro-inertia density

$k$-thermal conductivity of the fluid

$k_{c}$ - chemical reaction constant

$k_{p} \quad$ - permeability of the porous medium

$N$ - microrotation components normal to the $x y$ - plane

$T$ - temperature of the fluid

$T_{W} \quad$ - temperature of the fluid at the surface

$T_{\infty} \quad$ - temperature of the fluid outside the boundary layer

$u$ and $v$ - are velocity components along the $x$ and $y$ axes

$\beta_{T} \quad$ - coefficient of thermal expansion

$\beta_{C}-$ coefficient of concentration expansion

$\gamma-$ spin gradient viscosity

$\kappa_{v} \quad$ - micro-rotation viscosity or the vortex viscosity

$\mu$ - dynamic viscosity

$\rho-$ density of the fluid

$\sigma$ - electrical conductivity of the fluid

u - kinematic viscosity

\section{References}

[1] Eringen A.C. (1960): Theory of micropolar fluids. J. Math. Mech., vol.6, pp.1-18.

[2] Sakiadis B.C. (1961): Boundary layer behavior on continuous solid surfaces: II. The boundary layer on continuous flat surface. AIChE J., vol.7, pp.221-225.

[3] Crane L.J. (1970): Flow past a stretching plane. Z. Angew. Math. Phys., vol.21, pp.645-647.

[4] Nield D.A. and Bejan A. (1999): Convection in Porous Media. 2nd ed. New York: Springer.

[5] Lukaszewicz G. (1999): Micropolar Fluids: Theory and Applications. Boston: Birkhäuser.

[6] Srinivasacharya D., Mendu Upendar (2013): Effect of double stratification on MHD free convection in a micropolar fluid. Journal of the Egyptian Mathematical Society, vol.21, pp.370-378. 
[7] Ashwin Ramachandran, Bijaylakshmi Saikia, Krishnendu Sinha and Rama Govindarajan (2016): Effect of Prandtl number on the linear stability of compressible Couette flow. International Journal of Heat and Fluid Flow, pp.1-9.

[8] Asma Khalid, Ilyas Khan, Arshad Khan and Sharidan Shafie (2015): Conjugate transfer of heat and mass in unsteady flow of a micropolar fluid with wall couple stress. AIP ADVANCES 5, 127125.

[9] Sohail Nadeem, Sadaf Masood, Rashid Mehmood and Muhammad Adil Sadiq (2015): Optimal and Numerical Solutions for an MHD Micropolar Nanofluid between Rotating Horizontal Parallel Plates, PLOS ONE | DOI:10.1371/journal.pone.0124016.

[10] Das K. (2012): Slip effects on heat and mass transfer in MHD micropolar fluid flow over an inclined plate with thermal radiation and chemical reaction. Int. J. Numer. Meth. Fluids., vol.70, pp.96-113, DOI: 10.1002/fld.2683.

[11] Adhikari A. and Maiti A.K. (2014): MHD micropolar fluid flow towards a vertical surface in presence of heat. Journal of Imvi, vol.4, pp.1-25, DOI:11.7251/jimvi140101a.

[12] Habibi Matin M., Dehsara M. and Abbassi A. (2012): Mixed convection MHD flow of nanofluid over a non-linear stretching sheet with effects of viscous dissipation and variable magnetic field. ISSN 1392 - 1207. Mechanika, Vol.18, No.4, pp.415-423, http:// dx.doi.org/ 10.5755/ j01.mech.18.4.2334.

[13] Chaudhary R.C. and Jha A.K. (2008): Effect of chemical reaction on MHD micropolar fluid flow past a vertical plate in slip-flow regime. Appl. Math. Mech. -Engl. Ed., vol.29, No.9, pp.1179-1194. DOI 10.1007/s10483-0080907-x.

[14] Kashif Ali, Muhammad Farooq Iqbal, Zubair Akbar and Muhammad Ashraf (2014): Numerical simulation of unsteady water-based nanofluid flow and heat transfer between two orthogonally moving porous coaxial disks. Journal of Theoretical and Applied Mechanics, vol.52, No.4, pp.1033-1046.

[15] Syed Tauseef Mohyud-Din, Saeed Ullah Jan, Umar Khan and Naveed Ahmed (): MHD flow of radiative micropolar nanofluid in a porous channel: optimal and numerical solutions. Neural Comput. and Applic. DOI 10.1007/s00521-016-2493-3.

[16] El-Dabe N.T., Ghaly A.Y., Rizkallah R.R., Ewis K.M. and Al-Bareda A.S. (2015): Numerical solution of MHD flow of micropolar fluid with heat and mass transfer towards a stagnation point on a vertical plate. American Journal of Computational Mathematics, vol.5, pp.158-174. http://dx.doi.org/10.4236/ajcm.2015.52013.

[17] Srinivas Maripala and Kishan Naikoti (2016): MHD effects on micropolar nanofluid flow over a radiative stretching surface with thermal conductivity. Advances in Applied Science Research, vol.7, No.3, pp.73-82, ISSN: 0976-8610.

[18] Ali J. Chamkha, Mohamed R.A. and Sameh E. Ahmed (): Unsteady MHD natural convection from a heated vertical porous plate in a micropolar fluid with Joule heating, chemical reaction and radiation effects. Meccanica, DOI 10.1007/s11012-010-9321-0.

[19] Sandeep N., Sulochana C., Sugunamma V., Raju C.S.K. and Jayachandra Babu M. (2015): Unsteady boundary layer flow of thermophoretic MHD nanofluid past a stretching sheet with space and time dependent internal heat source/sink. Appl. Appl. Math. ISSN:1932-9466, vol.10, No.1, pp.312-327.

[20] Khedr M.E.M., Chamkha A.J. and Bayomi M. (2009): MHD flow of a micropolar fluid past a stretched permeable surface with heat generation or absorption. Nonlinear Analysis: Modelling and Control, vol.14, No.1, pp. $27-40$.

[21] Sandeep N. and Sulochana C. (2015): Dual solutions for unsteady mixed convection flow of MHD micropolar fluid over a stretching/shrinking sheet with non-uniform heat source/sink. Engineering Science and Technology, an International Journal, vol.18, 738e 745.

[22] Satya Narayana P.V., Venkateswarlu B. and Venkataramana S. (2013): Effects of Hall current and radiation absorption on MHD micropolar fluid in a rotating system. Ain Shams Engineering Journal, vol.4, pp.843-854.

[23] Kelson N.A. and Desseaux A. (2001): Effects of surface conditions on flow of a micropolar fluid driven by a porous stretching sheet. International Journal of Engineering Science, vol.39, pp.1881-1897.

[24] Ramana Reddy G.V., Bhaskar Reddy N. and Chamkha A.J. (2016): MHD mixed convection oscillatory flow over a vertical surface in a porous medium with chemical reaction and thermal radiation. Journal of Applied Fluid Mechanics, vol.9, No.3, pp.1221-1229. 
[25] Pal D. and Chatterjee S. (2015): Effects of radiation on Darcy-Forchheimer convective flow over a stretching sheet in a micropolar fluid with non- uniform heat source/sink. Journal of Applied Fluid Mechanics, vol.8, No.2, pp.207-212.

[26] Lakshmi R., Ramana Reddy G.V. and Jayarami Reddy K. (2015): Thermal radiation and variable viscosity on steady MHD free convective flow over a stretching sheet in Presence of heat source, dissipation and chemical Reaction. Global Journal of Pure and Applied Mathematics (GJPAM) ISSN 0973-1768, vol.11, No.2, pp.246281.(Scopus)

[27] Jhansi Rani K., Ramana Reddy G.V., Ramana Murthy Ch.V. and Ramana Murthy M.V. (2015): Heat and mass transfer effects on MHD free convection flow over an inclined plate embedded in a porous medium. Int. J. Chem. Sci., vol.13, No.4, pp.1998-2016.(Scopus)

[28] Ramana Reddy G.V., Bhaskar Reddy N. and Gorla R.S.R. (2016): Radiation and chemical reaction effects on MHD flow along a moving vertical porous plate. International Journal of Applied Mechanics and Engineering, vol.21, No.1, pp.157-168.

Received: October 3, 2016

Revised: December 16, 2017 